\title{
Design and Electromagnetic Analysis of a HTS Linear Synchronous Motor
}

\author{
Jianxun Jin, Luhai Zheng \\ Center of Applied Superconductivity and Electrical Engineering \\ University of Electronic Science and Technology of China \\ Chengdu, China \\ jxjin@uestc.edu.cn, zluhai@uestc.edu.cn
}

\author{
Youguang Guo, Jianguo Zhu \\ School of Electrical, Mechanical and Mechatronic Systems \\ University of Technology Sydney, Australia \\ Sydney, Australia \\ youguang@eng.uts.edu.au
}

\begin{abstract}
High temperature superconducting (HTS) linear synchronous motor (LSM) integrated with HTS magnetic levitation system, can realize self-levitation and self-guidance without any sliding friction, which will have important applications in many fields such as electromagnetic aircraft launch system, maglev transportation. This paper presents the design and electromagnetic analysis of a HTS LSM, which is levitated by a magnetic levitation system consisting of HTS bulkspermanent magnet (PM) guideways. Numerical analysis and magnetic field finite element analysis (FEA) methods are applied to analyze the thrust, levitation and guidance force characteristics, and the electromagnetic performance of HTS LSM under no-load and load situations are studied with the analysis results are given. Finally, the primary motor running testing results are provided.
\end{abstract}

Keywords-high temprature superconductor; HTS linear synchronous motor; electromagnetic characteristic;HTS magnetic levitation; HTS bulk

\section{INTRODUCTION}

A high temperature superconducting (HTS) bulk can trap higher magnetic field than a permanent magnet, and a few compact and higher-performance electric motors and linear actuators are being realized by using HTS bulks [1,2]. Now, the HTS linear synchronous motor (LSM) technology using bulk superconductor magnet (BSCM) has been developed [25]. Compared to the conventional linear motor, a HTS LSM has advantages, such as smaller volume, less weight, bigger propulsion, and higher power factor.

Since stable levitation force can be generated between NdFeB permanents magnets (PMs) and HTS bulks bathed in liquid nitrogen [6,7], HTS magnetic levitation system containing PMs and HTS bulks can be used in a HTS LSM to replace the conventional slide rail [8]. The main advantage of HTS magnetic levitation system is the strong levitating force with passive and self-stabilizing feature, leading to the removal of the sophisticated control system for regulating the air-gap between PM-guideway and levitated moving secondary, which is necessary for other types of levitation transportation systems. So that the HTS LSM integrated with the HTS magnetic levitation system can be applied in transportation field such as maglev, electromagnetic airplane launch system, and many other industrial automation control fields etc.

This work is supported by the Chinese High Technology Development Plan Project under Grant No. 2007AA03Z208.
In this paper, the design of a HTS LSM levitated using a HTS levitation system is presented. Numerical analyses for thrust, levitation and guidance force characteristics are conducted with results obtained. The electromagnetic characteristics of HTS LSM under no-load and load situations are studied with the analysis results are given by finite element analysis (FEA) method. Finally, the primary LSM running testing results are presented.

\section{HTS LSM MODEL}

The structure model of the designed HTS LSM is shown in Fig. 1. The primary stator and the secondary rotor of the HTS LSM are composed of three-phase copper windings and YBCO BSCMs, respectively, and the magnets are put in the cryogenic vessels with alternating poles of $\mathrm{N}$ and $\mathrm{S}$ in longitudinal direction. Table I lists the major dimensions and parameters of the HTS LSM.

The HTS magnetic levitation systems are used on both sides of the HTS LSM, and the structure of the PM-guideway is shown in Fig. 2, which is consisted of PMs and assembled magnetic iron (Fe). The magnetization directions of left-side and right-side PMs are along the vertical direction, and the middle PMs are installed with opposite magnetization direction. The ferromagnet put in the middle of PMs has the function of concentrating magnetic field, and generates strong magnetic field on the upper surface of PM-guideways and weak magnetic field on the lower surface.

The HTS magnetic levitation system can realize selflevitation and self-guidance without any assistant control equipment. So that the HTS LSM can run freely without any sliding friction force with stable air-gap length.

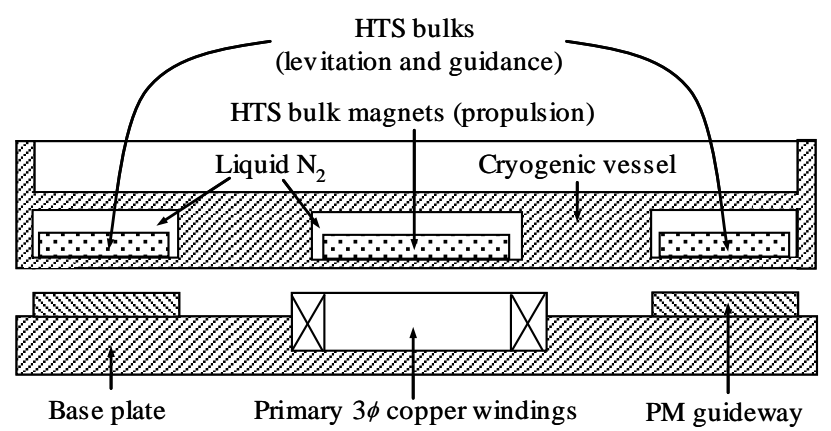

Figure 1. Model of HTS LSM levitated by HTS magnetic-levitation system. 


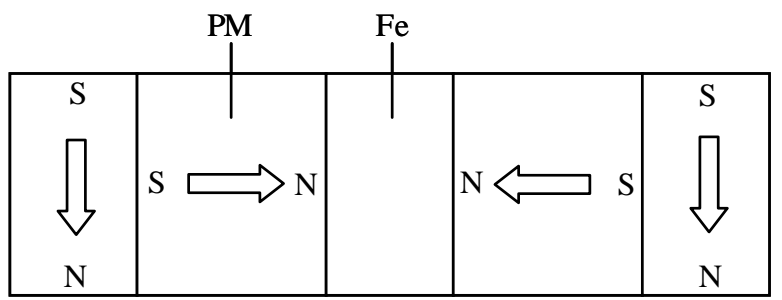

Figure 2. Structure of PM-guideway.

TABLE I. MAJOR DIMENSIONS AND PARAMETERS OF HTS LSM.

\begin{tabular}{|c|c|}
\hline Primary & \\
\hline \multicolumn{2}{|l|}{ Copper windings } \\
\hline Number of turns & 200 \\
\hline Diameter of copper wire (mm) & 1.18 \\
\hline Resistivity $(\Omega \cdot \mathrm{m})$ & 1.75E-008 \\
\hline \multicolumn{2}{|l|}{ Iron core } \\
\hline Tooth length (movement direction) (mm) & 10 \\
\hline Tooth width $l_{t}(\mathrm{~mm})$ & 150 \\
\hline Tooth depth (mm) & 100 \\
\hline Slot width (mm) & 20 \\
\hline Tooth pitch (mm) & 30 \\
\hline Pole pitch $\tau(\mathrm{mm})$ & 45 \\
\hline Main air gap $g(\mathrm{~mm})$ & 6 \\
\hline \multicolumn{2}{|l|}{ Secondary } \\
\hline \multicolumn{2}{|l|}{ BSCM } \\
\hline BSCM length (movement direction) $l_{s}(\mathrm{~mm})$ & 45 \\
\hline BSCM width $w_{s}(\mathrm{~mm})$ & 45 \\
\hline BSCM height $h_{s}(\mathrm{~mm})$ & 12 \\
\hline BSCM trapping field $B(\mathrm{~T})$ & 0.35 \\
\hline Number of BSCMs & 6 \\
\hline Back iron thickness (mm) & 10 \\
\hline \multicolumn{2}{|l|}{ Running parameters } \\
\hline Frequency (Hz) & 10 \\
\hline Phase voltage (V) & 165 \\
\hline Phase current (A) & 3.3 \\
\hline Phase resistance $(\Omega)$ & 27 \\
\hline Thrust force (N) & 363 \\
\hline
\end{tabular}

\section{NUMERICAL ANALYSIS OF HTS LSM}

\section{A. Thrust Force Analysis}

The transverse section and longitudinal section of the HTS LSM are shown in Fig. 3. The length of HTS bulks $l_{s}$ is equal to the pole pitch $\tau$ with no gap between them. Neglecting the magnetic resistance of primary iron-core and back-iron, the magnetic flux one coil applied by one pole-pairs of BSCMs as

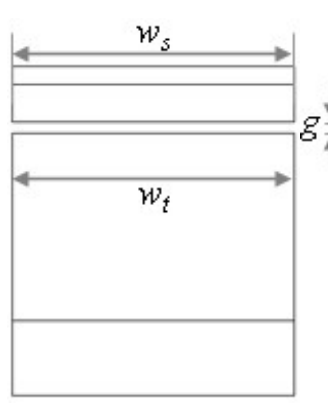

(a)

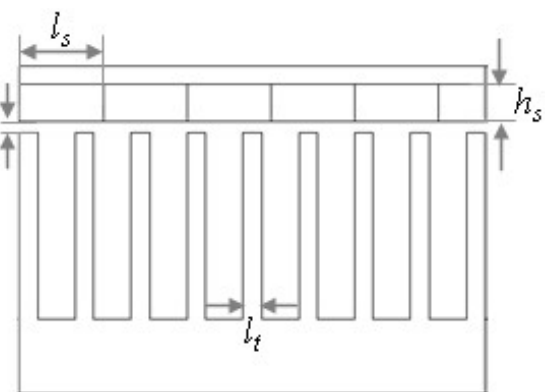

(b)
Figure 3. Model of HTS LSM (a) transverse section, (b) longitudinal section.

$$
\begin{aligned}
\psi_{\mathrm{BSCM}} & =\frac{\mathcal{F}}{R_{\mathrm{tot}}}=\frac{H_{c} \cdot h_{s}}{h_{s} / \mu_{r} \mu_{0} l_{s} w_{s}+g /\left[\mu_{0} \cdot\left(l_{s}+l_{t}\right) / 2 \cdot\left(w_{s}+w_{t}\right) / 2\right]} \\
& =\frac{\mu_{0} H_{c} h_{m} l_{m} w_{m}\left(l_{s}+l_{m}\right)\left(w_{m}+w_{s}\right)}{4 g l_{m} w_{m}+h_{m} l_{m} w_{m}+h_{m} l_{m} w_{s}+h_{m} l_{s} w_{m}+h_{m} l_{s} w_{s}}
\end{aligned}
$$

where $\mathscr{F}$ is the magnetic potential of magnetic circuit, $R_{\text {tot }}$ the total magnetic resistance, $R_{s}$ the magnetic resistance of BSCM, $R_{g}$ the magnetic resistance of air-gap, $\mu_{\mathrm{r}}$ the relative permeability of BSCM $\left(\mu_{\mathrm{r}}=1\right), H_{c}$ the magnetic field intensity of BSCM, $\mu_{0}\left(4 \pi \times 10^{-7} \mathrm{H} / \mathrm{m}\right)$ the permeability of vacuum; $l_{s}, w_{s}$, and $h_{s}$ the length, width and height of BSCM respectively; $l_{t}$, $w_{t}$ the length and width of stator-tooth respectively. When the secondary rotor moves, an electromotive force (EMF) is induced in the stator windings. By differentiating the BSCM flux of phase winding against time, the back EMF is determined with an rms value as

$$
E_{\mathrm{rms}}=\sqrt{2} \pi f N_{c} k_{N} \psi_{\mathrm{BSCM}}
$$

The electromagnetic power $P_{\mathrm{em}}$ is

$$
P_{\mathrm{em}}=3 \times E_{\mathrm{rms}} \times I_{1} \times \cos \varphi
$$

So the fundamental wave electromagnetic thrust force $F_{\mathrm{em}}$ is

$$
F_{\mathrm{em}}=\frac{p \times P_{\mathrm{em}}}{v}=\frac{p \times 3 \times \mathrm{NE}_{\mathrm{rms}} \times I_{1}}{2 \tau f}=\frac{3 p N_{c} k_{N} I_{1}}{\sqrt{2} \tau} \psi_{\mathrm{BSCM}}
$$

where $f$ is the frequency, $N_{c}$ the turns of phase winding, $k_{N}$ the winding factor $\left(k_{N}=1\right.$ when the diametrical-pitch concentrated windings are applied), $I_{1}$ the phase current, $\cos \varphi$ the power factor, $v$ the linear velocity, $\tau$ the pole pitch, $p$ the pole-pairs.

Fig. 4 shows the thrust force $F_{x}$ versus the thickness of BSCMs for various widths of bulk magnets. It is observed that the $F_{x}$ increases with the increases of the thickness of BSCM, and tends to saturation gradually. It also shows that the more the BSCM width increases, the bigger the $F_{X}$ is.

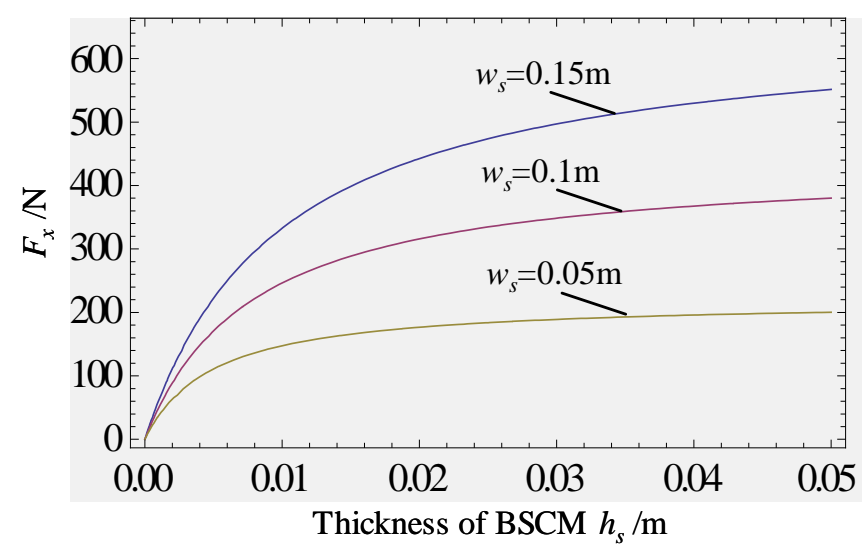

Figure 4. $F_{\mathrm{x}}$ versus $h_{s}$ for different widths of BSCMs 


\section{B. Levitation Force and Guidance Force Analysis}

The levitation force and guidance force are the product of an external magnetic field and a shielding current flowing in a HTS bulk, and is calculated by the following equations

$$
\begin{gathered}
F_{\mathrm{Lev}}=\int_{0}^{T H} \int_{L / 2}^{L / 2-\delta} \int_{W / 2}^{W / 2-\delta} J_{c} \times B_{x} \mathrm{~d} x \mathrm{~d} y \mathrm{~d} z \\
F_{\text {Gui }}=\int_{0}^{T H} \int_{L / 2}^{L / 2-\delta} \int_{W / 2}^{W / 2-\delta} J_{c} \times B_{z} \mathrm{~d} x \mathrm{~d} y \mathrm{~d} z
\end{gathered}
$$

where $F_{\mathrm{Lev}}$ is the levitation force, $F_{\mathrm{Gui}}$ the guidance force, $J_{c}$ the critical current density, $B_{X}$ the field along the transverse direction, $B_{z}$ the field along the vertical direction, $\delta$ the depth of field penetration. Here, it is assumed that induced shielding currents flowing in the bulk body were equal to the critical currents. The $\delta$ used as integral range is given by the following relation [9]

$$
\delta=\frac{B_{z}-B_{\mathrm{zfc}}}{\lambda \mu_{0} J_{c}}
$$

where $B_{\mathrm{zfc}}$ is the trapped magnetic field, $\lambda$ the Nagaoka coefficient determined by the configuration of a sample. The Fig. 5 shows the interaction between a HTS bulk and the PMguideway, and the Fig. 6 and Fig. 7 are the distributions of $B_{x}$ and $B_{z}$ for different heights $h$ from upper surface of primary stator, respectively.

The relation shape between the levitation force and the distance between a HTS bulk and the PM-guideway can be described with an exponential function as follows [10]

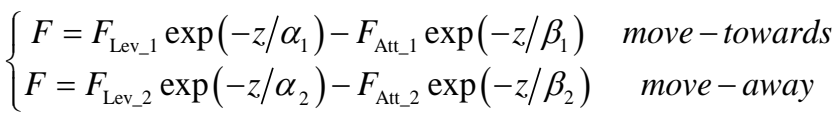

where $F_{\text {Lev_1 }}$ and $F_{\text {ATT_1 }}$ are the levitation force and attractive force when HTS bulk is moved towards the PM-guideway, respectively; $F_{\text {Lev_2 }}$ and $F_{\text {ATT_2 }}$ are the levitation force and attractive force when HTS bulk is moved away the PMguideway, respectively; $\alpha_{1}, \alpha_{2}, \beta_{1}$ and $\beta_{2}$ are constants, which are closely related to the superconductor and the distribution of magnetic field; $z$ the distance between the HTS bulk and PMguideway. Fig. 8 shows the levitation force of a HTS bulk above the PM-guideway.

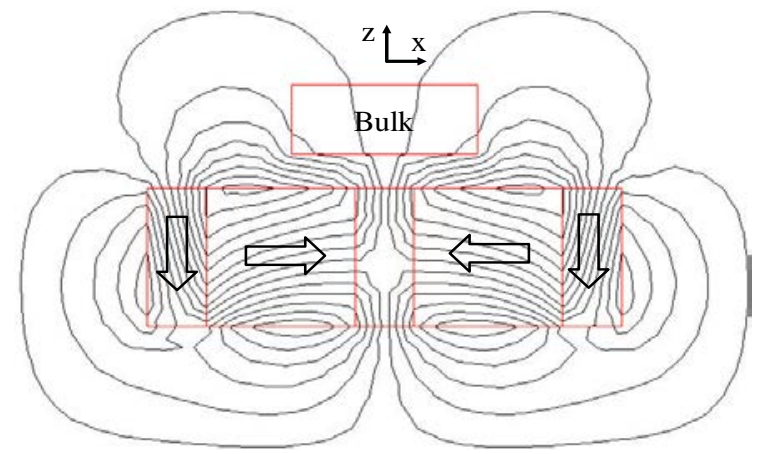

Figure 5. Model of interaction between a HTS bulk and the PM-guideway.

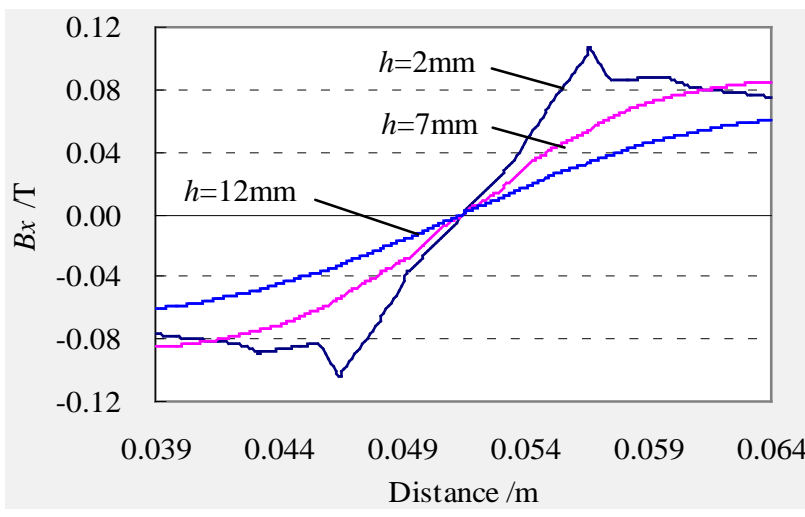

Figure 6. Distributions of $B_{\mathrm{x}}$ for different heights.

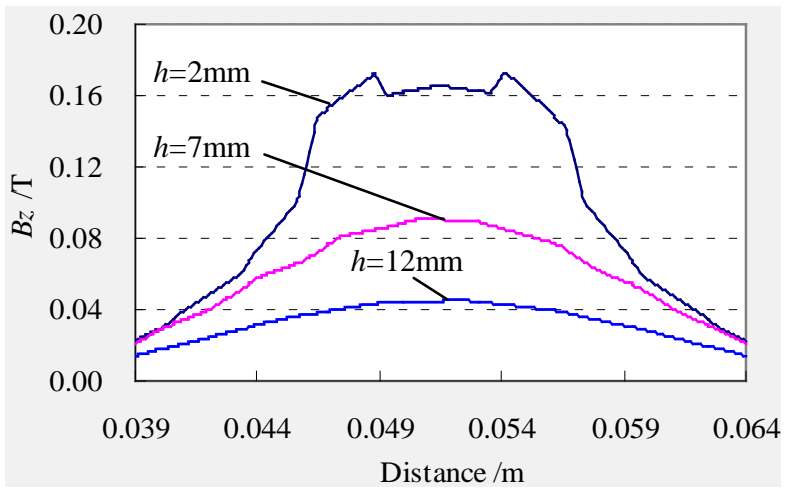

Figure 7. Distributions of $B_{\mathrm{z}}$ for different heights

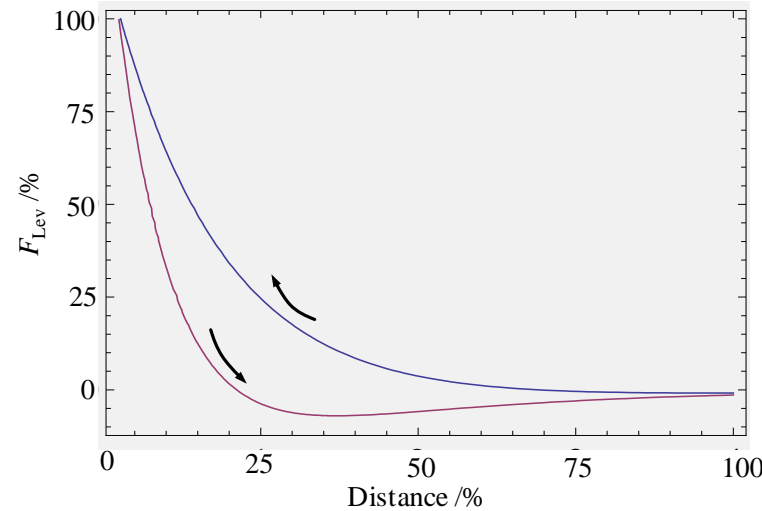

Figure 8. The levitation force between a HTS bulk and the PM-guideway.

\section{ELECTROMAGNETIC CHARACTERISTIC ANALYSIS OF HTS LSM}

According to the above assumptions and motor geometric parameters, 2-D finite element model for the whole HTS LSM is built by the way of direct modeling method, which is sufficient for the theoretical analysis with relatively low computational complexity, and the end effect can also be considered.

The finite element model is shown in Fig. 9. The materials in model are numbered as: (1) air; (2) BSCM North (with the magnetization direction along the negative $y$-axis direction); (3) BSCM South (with the magnetization direction along the $y$ - 
axis direction): (4) BSCM back iron; (5) stator iron core; and (6) copper stranded coils. The magnetic vector potentials obey the periodical boundary conditions on the $y$-direction boundary lines of the primary stator and secondary rotor and the open middle air-gap line, and the time-stepping FEA method is applied in resolving. To simplify the analysis, the BSCM is modeled as a PM. The analysis results of no-load and load conditions of the HTS LSM are detailed as follows.

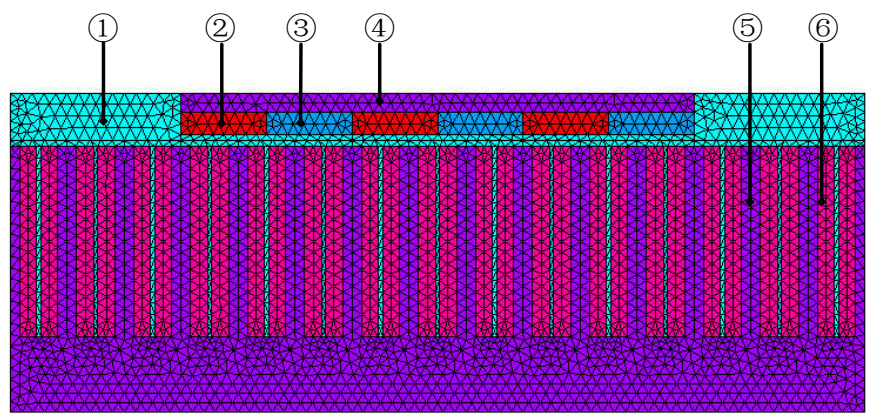

1. Air, 2. BSCM North, 3. BSCM South, 4. Back iron, 5. Iron core, 6. Coils.

Figure 9. Finite element model of the HTS LSM.

\section{A. No-Load Characteristics}

The distribution of magnetic flux density $B_{x}, B_{y}$ (along the longitudinal direction), and $B_{\text {sum }}$ (the vector sum) in middle airgap at no-load is shown in Fig. 10.

The BSCM flux, defined as the flux of a phase winding produced by the mover BSCMs, can be determined from the magnetic field distribution at no-load. The flux waveform can be calculated by moving the rotor for one pole-pair pitch in several steps. To increase the computational accuracy and efficiency, the stator and rotor are separately meshed and keep unchanged when the rotor moves. After the meshed rotor moves to a new position, the meshes are stitched with those of the stator along the middle air-gap. For the time-stepping analysis, the meshing of middle air-gap line is controlled such that the nodes of the stator and rotor sides coincide at each step [8].

Fig. 11 shows the magnetic flux lines distribution when the rotor has moved one pitch $\tau$ from the position $x=0$, and Fig. 12 is the BSCM flux linkage characteristics one coil for various lengths of air-gap. It is showed that the flux waveform is an almost perfect sinusoid, and the flux decreases with the increase of the air-gap length.

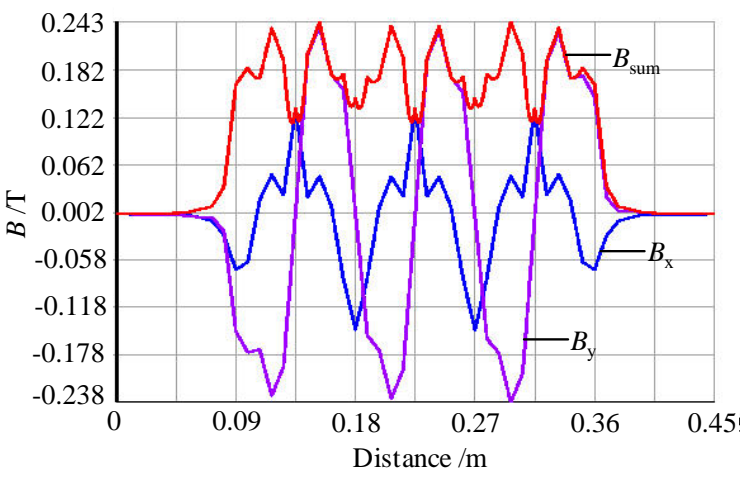

Figure 10. Distributions of $B_{x}, B_{y}$ and $B_{\text {sum }}$ along middle air-gap at no-load.

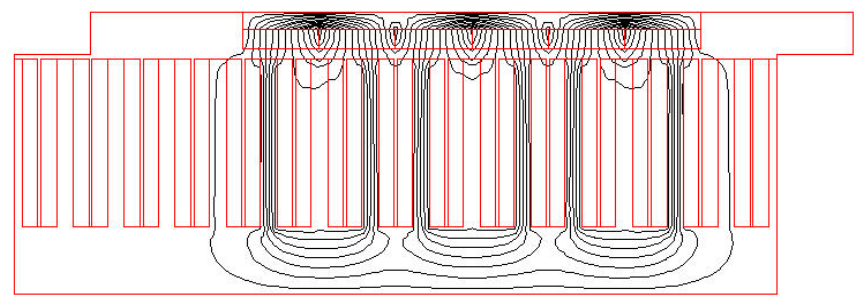

Figure 11. Distribution of magnetic flux lines when $\Delta x=\tau$.

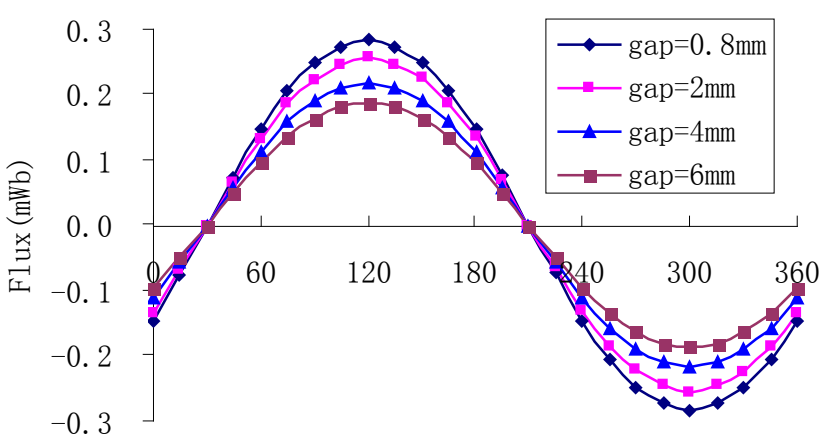

Mover position(ele. deg.)

Figure 12. BSCM flux linkage characteristics one coil for various air-gap length.

\section{B. Load Manetic Field Distribution}

The time-stepping simulation parameters as follows: the frequency $f=10 \mathrm{~Hz}$, the simulation time is one period of $T=0.1$ $\mathrm{s}$, the three-phase voltage: $U_{a}=165 \times \sin (\omega t), U_{b}=165 \times \sin (\omega t$ $2 \pi / 3), U_{c}=165 \times \sin (\omega t-4 \pi / 3)$. The solving is divided into 13 steps. In addition to the initial load step, the time period of every load step is $0.00833 \mathrm{~s}$, and each step has 10 substeps with the time step size of $0.000833 \mathrm{~s}$. Because it is not allowed to set time to zero in an ANSYS analysis, a very small time value of 0.1E-006 $\mathrm{s}$ is specified for the initial load step.

The distribution of magnetic flux lines in LSM at load is shown in Fig. 13, and Fig. 14 shows the magnetic field distribution in middle air-gap along transverse-direction and longitudinal-direction and the vector sum. As is indicated in the graph, the amplitude of magnetic flux density in air-gap is bigger than that at no-load.

Fig. 15 shows the $B_{\text {sum }}$ in air-gap changes with the time for different air-gap heights from upper-surface of primary stator. It is observed that the field waveforms are sinusoid, and the amplitude decreases with the increases of the height. It indicates that the shorter the air-gap length is, the bigger the electromagnetic force of the rotor will be suffered.

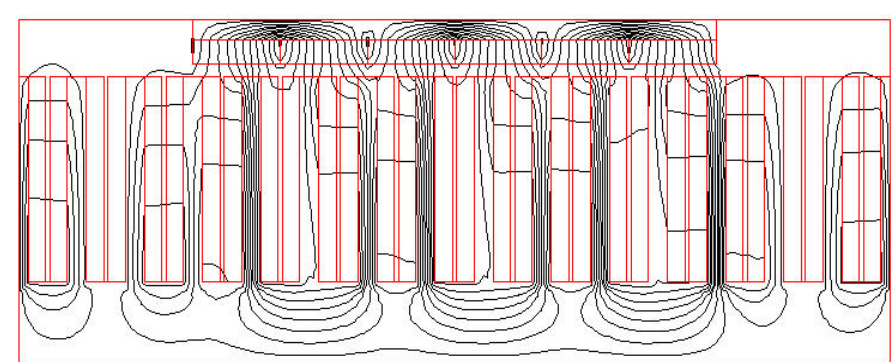

Figure 13. Distribution of magnetic flux lines at load condition. 


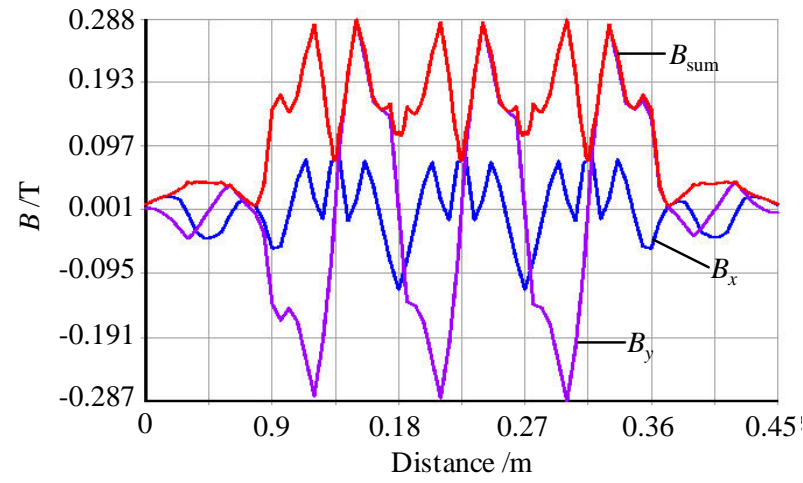

Figure 14. Distributions of $B_{x}, B_{y}$ and $B_{\text {sum }}$ along middle air-gap at load.

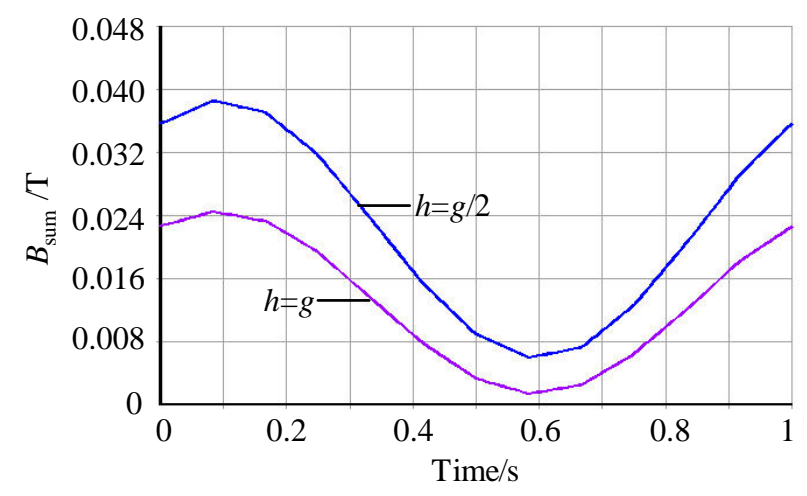

Figure 15. $B_{x}$ vs. time for different air-gap heights.

\section{Thrust Force Characteristics}

The thrust force is directly related to the trapped magnetic field, pole-pairs and length of HTS bulks, phase current of primary windings, thickness of back-iron, and length of air-gap and so on. The relationships are calculated and the simulation results are given as follows.

The thrust force $F_{X}$ versus phase current $I$ for different trapped magnetic field $B_{\text {trr }}$ of BSCMs is shown in Fig. 16. As can be seen from the chart, the $F_{X}$ increases approximately linear with the increase of the $I$ and the $B_{\text {trr }}$ of BSCMs, and the $F_{X}$ can reach $1000 \mathrm{~N}$ with $I=12 \mathrm{~A}(\mathrm{NI}=240 \mathrm{At})$ and $B_{\mathrm{trr}}=0.35 \mathrm{~T}$, or $I=5 \mathrm{~A}(N I=1000 \mathrm{At})$ and $B_{\mathrm{trr}}=0.6 \mathrm{~T}$, or $I=3 \mathrm{~A}(N I=600 \mathrm{At})$ and $B_{\text {trr }}=1 \mathrm{~T}$, or $I=2.5 \mathrm{~A}(N I=500 \mathrm{At})$ and $B_{\mathrm{trr}}=2 \mathrm{~T}$ when the other parameters based on Table 1.

Fig. 17 shows the $F_{x}$ versus the amount of BSCMs, which indicates that the $F_{X}$ increases proportionally to the amount of HTS bulk magnets. The length of BSCM also has important influence on $F_{X}$ as can be seen from Fig. 18. As is indicated in the graph, LSM will have relative bigger $F_{X}$ when the ratio of the length of BSCM to the pole pitch $\tau$ is between about 4:5 and about $1: 1$, which will be the important reference for the design of a HTS LSM.

Fig. 19 and Fig. 20 show that the $F_{x}$ and normal force $F_{y}$ versus the length of air-gap for different thickness of back-iron $H_{\text {back }}$, respectively. It is observed that $F_{x}$ and $F_{y}$ decrease with the increase of the length of air-gap, and increase with the increase of the thickness of back-iron when $H_{\text {back }}$ smaller than $10 \mathrm{~mm}$. Fig. 21 shows the $F_{x}$ versus the thickness of BSCM for various lengths of air-gap. The results indicate that the appropriate thickness of BSCMs and back-iron and the length of air-gap should be selected to get an optimal $F_{x}$ performance of HTS LSM.

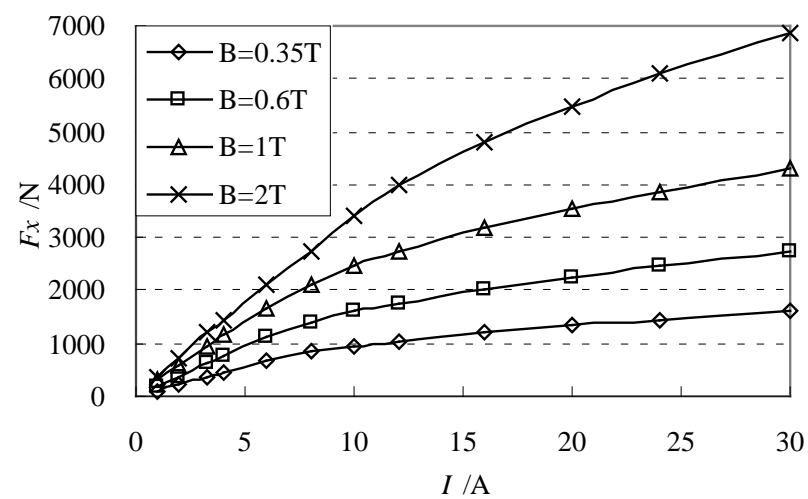

Figure 16. $F_{x}$ vs. the current for different trapped magnetic field of BSCMs.

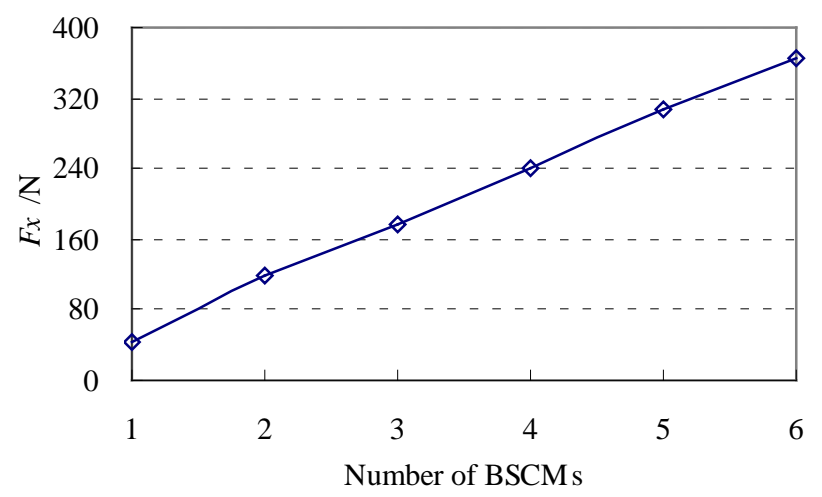

Figure 17. $F_{X}$ vs. the amount of BSCMs.

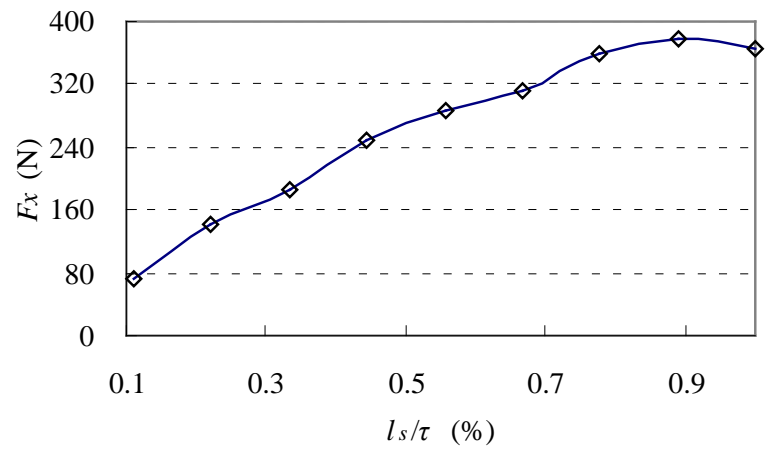

Figure 18. $F_{X}$ vs. the length of BSCMs.

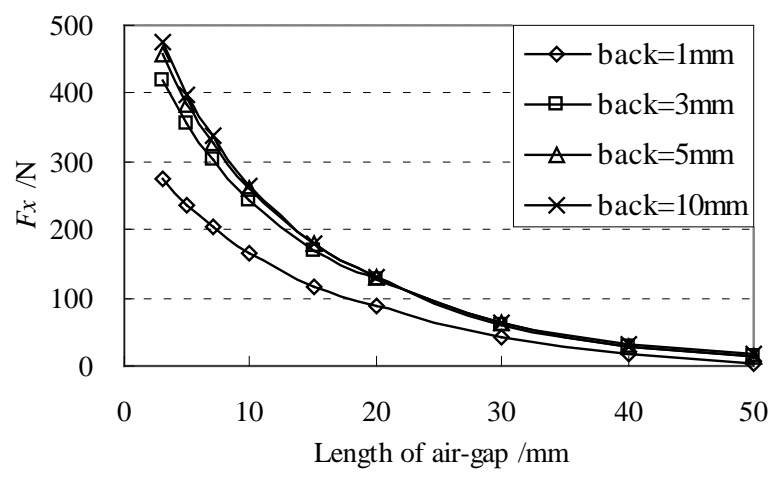

Figure 19. $F_{x}$ vs. the length of air-gap for different thicknesses of back-iron. 


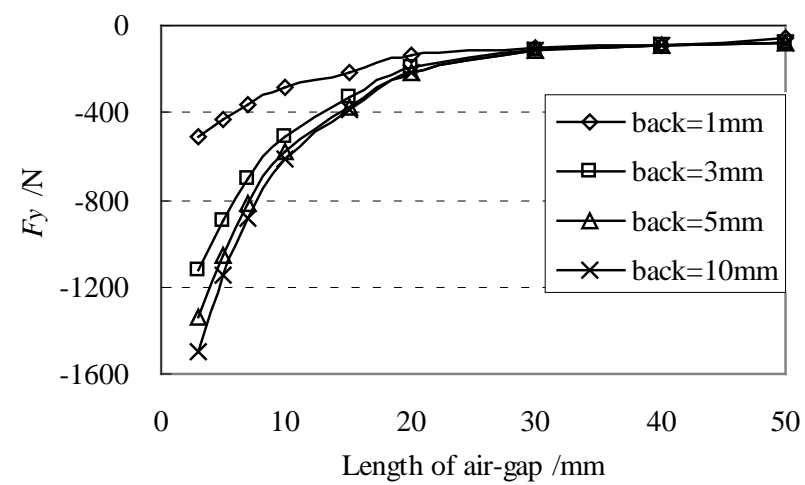

Figure 20. $F_{y}$ vs. the length of air-gap for different thicknesses of back-iron.

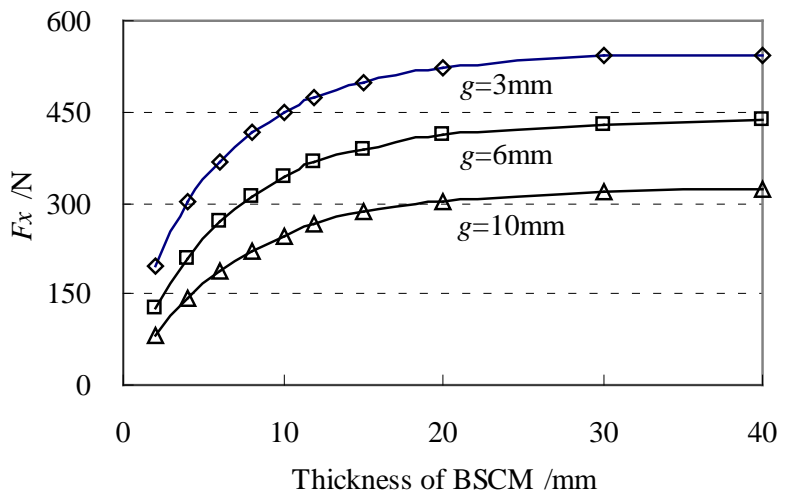

Figure 21. $F_{X}$ vs. the thickness of BSCM for various lengths of air-gap.

\section{TESTING Results}

The HTS LSM experimental device is shown in Fig. 22, which is composed of primary stator and secondary mover, and the secondary BSCMs are built in a cryogenic vessel fixed on the secondary mover, which can slide freely along the guide rails on both sides of the stator. The experimental slide rail will be replaced by the HTS levitation system.

The converter based on the Voltage Space Vector PWM (SVPWM) strategy is applied to control the running of HTS LSM. Fig. 23 shows the $F_{x}$ versus the frequency for different air-gap lengths. It indicates that the $F_{X}$ decrease slightly with the increase of the frequency, and the length of air-gap has great influence on $F_{x}$. Therefore the appropriate air-gap length should be selected by considering the practical requirements.

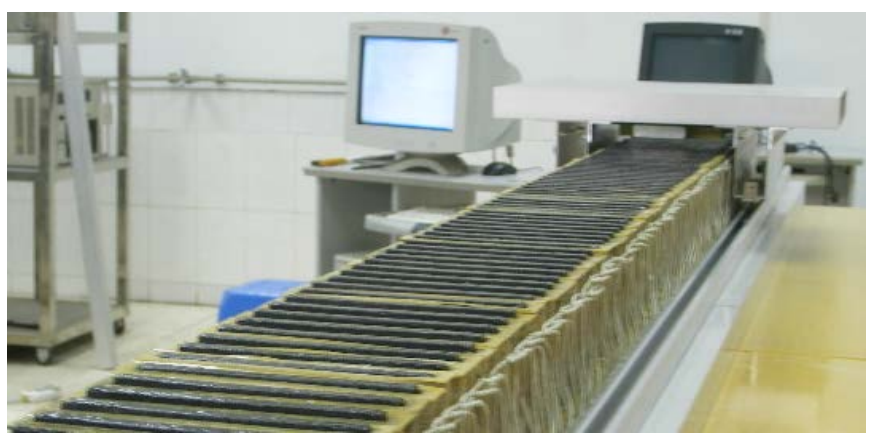

Figure 22. Experimental setup of the HTS LSM.

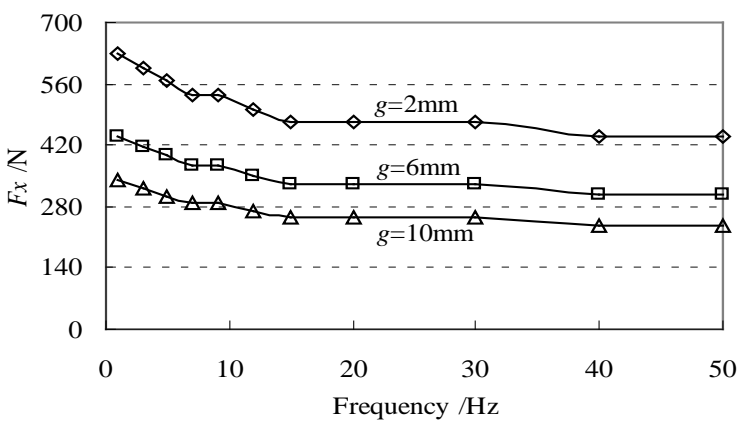

Figure 23. $F_{x}$ versus frequency for different air-gap length.

\section{CONCLUSION}

The structure design of new-type HTS LSM levitated by HTS levitation system has been presented. The numerical models of thrust, levitation and guidance force were built. The electromagnetic characteristics of HTS LSM were studied. The results obtained show that (i) when the trapped magnetic field of BSCM increases to $2 \mathrm{~T}$ from $0.35 \mathrm{~T}$, the $F_{x}$ will increase by 3.3 times, which can't be realized by using conventional permanent magnets; (ii) when the length of BSCMs are located between $0.8 \tau$ and $\tau$, the LSM will have better $F_{x}$ performance; (iii) the $F_{x}$ increases with increasing the thickness of BSCM when it is less than $20 \mathrm{~mm}$; (iv) when the thickness of backiron over $10 \mathrm{~mm}$, the influence on $F_{x}$ becomes saturated. Based on the above analysis, the HTS LSM can be optimized with HTS bulks and achieves better performance.

\section{REFERENCES}

[1] J. X. Jin, L. H. Zheng, Y. G. G, and J. G. Zhu, "Development of High Temperature Superconducting Machines,” Journal of Japan Society of Applied Electromagnetics and Mechanics, vol. 15(s), pp. S88-S91, 2007.

[2] G. Stumberger, M. T. Aydemir, and A. L. Thomas, "Design of a linear bulk superconductor magnet synchronous motor for electromagnetic aircraft launch systems,” IEEE Trans. Appl. Super., vol. 14, no. 1, pp. 54-62, 2004.

[3] J. X. Jin, and L. H. Zheng, "Performance analysis and optimization design of a HTS LSM,” Journal of Science, Technology and Engineering, vol. 2, no. 1, pp. 19-22, 2008.

[4] A. Takahashi, H. Ueda, and A. Ishiyama, "Trial production and experiment of linear synchronous actuator with field-cooled HTS bulk secondary,” IEEE Trans. Appl. Super., vol. 13, no. 2, pp. 2251-2254, 2003.

[5] K. Yoshida and H. Matsumoto, "Design and simulation of HTS bulk linear synchronous motor,” Physica C, vol. 378-381, pp. 833-837, 2002.

[6] J. X. Jin, "High $T_{c}$ superconductor theoretical models and electromagnetic flux characteristics,” Journal of Electronic Science and Technology of China, vol. 4, no. 3, pp. 202-208, September 2006.

[7] J. X. Jin and L. H. Zheng, "Verification of levitation force between a HTS and a permanent magnet,” Physica C, vol. 460-462, pp. 1457-1458, 2007

[8] Y. G. Guo, J.X. Jin, L.H. Zheng, J.G. Zhu, and H.Y. Lu, “A permanent magnet linear synchronous motor drive for HTS maglev Transportation Systems, ” Journal of Electronic Science and Technology of China, vol .6, no. 2, pp. 125-129, June 2008.

[9] T. Suzuki, S. Araki, K. Koibuchi, K. Ogawa, K. Sawa, K. Takeuchi, et al, "A study on levitation force and its time relaxation behavior for a bulk superconductor-magnet system,” Physica C, vol. 468, no. 15-20, pp. 1461-1464, 2008.

[10] F. C. Moon, K. C. Weng, and P. Z. Chang, "Dynamic magnetic forces in superconducting ceramics,” J. Appl. Phys., vol. 66, pp. 5643-5645, 1989. 